\title{
Universal Bounds on the Derivatives of the Symbol Error Rate for Arbitrary Constellations
}

\author{
Berkan Dulek, Member, IEEE
}

\begin{abstract}
The symbol error rate (SER) of the minimum distance detector under additive white Gaussian noise is studied in terms of generic bounds and higher order derivatives for arbitrary constellations. A general approach is adopted so that the recent results on the convexity/concavity and complete monotonicity properties of the SER can be obtained as special cases. Novel universal bounds on the SER, which depend only on the constellation dimensionality, minimum and maximum constellation distances are obtained. It is shown that the sphere hardening argument in the channel coding theorem can be derived using the proposed bounds. Sufficient conditions based on the positive real roots (with odd multiplicity) of an explicitly-specified polynomial are presented to determine the signs of the SER derivatives of all orders in signal-to-noise ratio. Furthermore, universal bounds are given for the SER derivatives of all orders. As an example, it is shown that the proposed bounds yield a better characterization of the SER for arbitrary two-dimensional constellations over the complete monotonicity property derived recently.
\end{abstract}

Index Terms-Completely monotone, Gaussian noise, higher order derivatives, maximum likelihood detection, symbol error rate (SER), universal bounds.

\section{INTRODUCTION}

$\mathbf{I}$ $\mathrm{N}$ digital communications, an important performance measure is the symbol error rate (SER). The SER of the minimum distance detector operating over the additive white Gaussian noise (AWGN) channel has long been a focus of research. In this endeavor, a number of exact and approximate analytical results that characterize the SER of various modulation formats over nonfading and fading AWGN channels are obtained [1]-[3]. Recently, some generic properties of the SER that hold for arbitrary signal constellations impaired by AWGN have been identified [4]-[7]. In [4], the SER is shown to be convex in signal-to-noise ratio (SNR) for all one- and two-dimensional (1-D and 2-D, respectively) constellations. Concavity in higher dimensions at low to intermediate SNR is possible, while convexity is always guaranteed at high SNR with an explicitly-determined threshold, which depends only on the constellation minimum distance and dimensionality. Lower and upper bounds on the first and second derivatives of the SER in SNR are also derived. In the follow-up paper [5], the authors have provided tighter SNR thresholds to identify

Manuscript received June 18, 2013; revised October 09, 2013; accepted December 17, 2013. Date of publication December 23, 2013; date of current version February 06, 2014. The associate editor coordinating the review of this manuscript and approving it for publication was Prof. Emmanuel Candes.

The author is with the Department of Electrical and Electronics Engineering, Bilkent University, Bilkent, Ankara 06800, Turkey (e-mail: berkandulek@gmail.com).

Digital Object Identifier 10.1109/TSP.2013.2296273 the concavity/convexity regions, and extended the earlier results to a more general class of decoders under unimodal and spherically invariant noise. In [6], the SER of the minimum distance detector impaired by AWGN is found to be a completely monotone (c.m.) function of the SNR if the constellation matrix has a rank of one or two. ${ }^{1}$ This result is stronger than convexity because it also implies that all the successive derivatives of the SER alternate in sign. As an application of this property, it is shown that the average SERs of an arbitrary 1-D or 2-D constellation over two different fading AWGN channels can be compared using the stochastic Laplace transform order. In higher dimensions (3-D and up), it is proved in [7] that the SER can be characterized as the product of a c.m. function with a nonnegative power of SNR. However, whether the complete monotonicity property holds or not for higher dimensional constellations is left as an open problem.

In this paper, further results on the SER of the minimum distance detector are derived for arbitrary constellations under AWGN. In particular, the SER is characterized in terms of generic bounds and its higher order derivatives by exploiting the spherical symmetry of the Gaussian noise distribution. This information can provide valuable insights in the design and analysis of communications systems such as a better understanding of the constellation-independent system properties and an efficient implementation of the numerical optimization algorithms. A general approach is developed in this paper, so that the corresponding results presented in [4]-[6] appear as special cases. The main contributions are summarized as follows:

- Universal lower and upper bounds on the SER are obtained. The bounds depend only on the constellation dimensionality, minimum and maximum constellation distances. They cannot be improved without additional assumptions on the constellation geometry. It is also shown that the sphere hardening argument in the channel coding theorem can be derived using the proposed bounds.

- A general expression for the $k$ th derivative of the SER conditioned on the $i$ th symbol of the constellation being transmitted is presented $(k \geq 1)$. Based on this representation, sufficient conditions are provided to determine the signs of the SER derivatives of all orders over various SNR ranges for arbitrary constellations. More explicitly, sufficient conditions are governed by the positive real roots (with odd multiplicity) of an explicitly-specified polynomial. It is shown that the convexity/concavity and complete monotonicity properties of the SER established in [5,

\footnotetext{
${ }^{1}$ This includes all 1-D and 2-D constellations as well as any multidimensional constellation which is unitarily equivalent to a constellation whose points lie on a line or plane [6].
} 
Th. 2] and [6, Th. 1] for arbitrary constellations can be obtained as special cases. As another example, the SNR regions over which the third SER derivative is positive (or negative) are identified for all values of the constellation dimensionality. Simpler bounds are also proposed to determine the high SNR region, where the sign of the $k$ th derivative is given by that of $(-1)^{k}$.

- Universal lower and upper bounds are obtained for the SER derivatives of all orders. These bounds depend only on SNR, constellation dimensionality and order of the derivative. It is shown that the lower and upper bounds for the $k$ th derivative decrease in magnitude at least as the $k$ th power of the SNR up to a multiplicative constant. The proportionality constants for the lower and upper bounds can be computed via an explicitly-specified procedure. As an example, the bounds are stated explicitly in the case of arbitrary 2-D constellations. This is a significant improvement over the complete monotonicity property of the SER derived in $[6$, Th. 1]. Furthermore, the bounds given in [4, Th. 7] for the second derivative of the SER are corrected.

Notation: Throughout this paper, vectors and matrices are denoted by boldface lower and upper case letters, respectively. The superscript $(\cdot)^{T}$ denotes the transpose operator. The identity matrix is denoted by $\mathbf{I}$. $|\mathbf{x}|$ denotes the $\ell^{2}$-norm of the vector $\mathbf{x}$, i.e., $|\mathbf{x}|=\sqrt{\mathbf{x}^{T} \mathbf{x}}$. For scalar $x,|x|$ denotes the absolute value, and $\lfloor x\rfloor$ denotes the largest integer not greater than $x$. The set of real numbers is denoted by $\mathbb{R}$, and the set of positive integers (i.e., natural numbers) is denoted by $\mathbb{N}_{>0}$. The multivariate real Gaussian distribution with mean vector $\boldsymbol{\mu}$ and covariance matrix $\mathrm{C}$ is denoted by $\mathcal{N}(\boldsymbol{\mu}, \mathbf{C})$. The probability distribution function (PDF) of the random variable (RV) $X$ is denoted by $f_{X}(\cdot)$. The expectation of the function $g(\cdot)$ over the PDF of the RV $X$ is denoted by $\mathbb{E}\{g(X)\}$. The probability of correct decision, given that the $i$ th symbol of the constellation is transmitted, is denoted by $P_{c, i}(\rho)$ as a function of the SNR $\rho$. Likewise, the SER conditioned on the $i$ th symbol of the constellation being transmitted is denoted by $P_{e, i}(\rho) . P_{e}(\rho)$ denotes the SER averaged over the constellation points. The $k$ th derivative of the SER (the conditional SER) is denoted by $P_{e}^{(k)}(\rho)\left(P_{e, i}^{(k)}(\rho)\right)$ for all $k \in \mathbb{N}_{>0}$. $\operatorname{Pr}[\cdot]$ denotes the probability of the event inside the square brackets.

\section{SYSTEM MODEL}

We consider the standard baseband discrete-time system model for $M$-ary communications through AWGN, which is described as [4], [5], [7]

$$
\mathbf{y}=\mathbf{s}+\mathbf{z},
$$

where the transmitted symbol $\mathbf{s} \in \mathbb{R}^{N}$ is drawn from a constellation $\mathcal{S}:=\left\{\mathbf{s}_{1}, \ldots, \mathbf{s}_{M}\right\}$ and $N$ is the constellation dimensionality. The noise is assumed to be $\mathbf{z} \sim \mathcal{N}\left(\mathbf{0}, \sigma_{0}^{2} \mathbf{I}\right)$, where $\sigma_{0}^{2}$ is the noise variance per dimension. More explicitly, the PDF of the noise $\mathbf{z}$ is

$$
f_{\mathbf{z}}(\mathbf{x})=\left(\frac{1}{2 \pi \sigma_{0}^{2}}\right)^{\frac{N}{2}} e^{-\frac{|\mathbf{x}|^{2}}{2 \sigma_{0}^{2}}} .
$$

Assuming that the average symbol energy is normalized as $M^{-1} \sum_{i=1}^{M}\left|\mathbf{s}_{i}\right|^{2}=1$, the average SNR is defined as $\rho:=1 / \sigma_{0}^{2}$. At the receiver, the maximum likelihood (ML) detector is con- sidered. Since ML detector under AWGN is the minimum distance one [2], the detected symbol $\hat{s}$ is given by

$$
\hat{\mathbf{s}}=\underset{\mathbf{s} \in \mathcal{S}}{\arg \min }|\mathbf{y}-\mathbf{s}| .
$$

Assuming that the origin is shifted to the constellation point $\mathbf{s}_{i}$, the decision region (Voronoi region) of $\mathbf{s}_{i}$, denoted by $\Omega_{i}$, is given by

$\Omega_{i}=\left\{\mathbf{x} \in \mathbb{R}^{N} \mid \mathbf{a}_{i, j}^{T} \mathbf{x} \leq b_{i, j}, j=1, \ldots, i-1, i+1, \ldots, M\right\}$,

where $\mathbf{a}_{i, j}=\left(\mathbf{s}_{j}-\mathbf{s}_{i}\right) /\left|\mathbf{s}_{j}-\mathbf{s}_{i}\right|$, and $b_{i, j}=\left|\mathbf{s}_{j}-\mathbf{s}_{i}\right| / 2$. Since an intersection of $M-1$ half-spaces is defined in (4), $\Omega_{i}$ is a convex set [8]. It is noted that the decision region does not depend on $\rho$. Furthermore, the minimum (maximum) distance from the origin to the boundary of the decision region $\Omega_{i}$ is defined as $d_{m i n, i}\left(d_{\max , i}\right)$. We also define $d_{\min }:=\min _{i}\left\{d_{\min , i}\right\} \quad\left(d_{\max }:=\max _{i}\left\{d_{\max , i}\right\}\right)$. It is noted that $d_{\min , i}=\min _{\mathbf{s}_{j} \in \mathcal{S}, \mathbf{s}_{j} \neq \mathbf{s}_{i}}\left|\mathbf{s}_{i}-\mathbf{s}_{j}\right| / 2$ and $d_{\text {min }}=\min _{\mathbf{s}_{i}, \mathbf{s}_{j} \in \mathcal{S}, \mathbf{s}_{i} \neq \mathbf{s}_{j}}\left|\mathbf{s}_{i}-\mathbf{s}_{j}\right| / 2$.

The probability of correct decision $P_{c, i}(\rho)$, conditioned on $\mathbf{s}=\mathbf{s}_{i}$ being transmitted, is given by [2]

$$
P_{c, i}(\rho)=\left(\frac{\rho}{2 \pi}\right)^{\frac{N}{2}} \int_{\Omega_{i}} e^{-\frac{\rho|\mathbf{x}|^{2}}{2}} \mathrm{~d} \mathbf{x} .
$$

Likewise, the conditional SER, $P_{e, i}(\rho)$ given that $\mathbf{s}=\mathbf{s}_{i}$ is transmitted, is obtained by

$$
P_{e, i}(\rho)=\left(\frac{\rho}{2 \pi}\right)^{\frac{N}{2}} \int_{\mathbb{R}^{N} \backslash \Omega_{i}} e^{-\frac{\rho|\mathbf{x}|^{2}}{2}} \mathrm{~d} \mathbf{x},
$$

where $\mathbb{R}^{N} \backslash \Omega_{i}=\left\{\mathbf{x} \in \mathbb{R}^{N} \mid \mathbf{x} \notin \Omega_{i}\right\}$. Clearly, $P_{e, i}(\rho)=1-$ $P_{c, i}(\rho)$. The probability of error averaged over all constellation points is

$$
P_{e}(\rho)=\sum_{i=1}^{M} \operatorname{Pr}\left[\mathbf{s}=\mathbf{s}_{i}\right] P_{e, i}(\rho)=1-P_{c}(\rho),
$$

where $\operatorname{Pr}\left[\mathbf{s}=\mathbf{s}_{i}\right]$ denotes the a priori probability of transmitting $\mathbf{s}_{i}$.

Next, an additional remark about the dimension of the constellation is noted. Let the $N$-by- $M$ constellation matrix corresponding to $\mathcal{S}$ be defined as $\mathbf{S}:=\left[\mathbf{s}_{1}, \ldots, \mathbf{s}_{M}\right]$, and the rank of $\mathbf{S}$ be denoted by $N^{*}$. It is shown in [7] that for any $M$-ary signal constellation $\mathcal{S}$ in $\mathbb{R}^{N}$, an equivalent $M$-ary signal constellation $\mathcal{S}^{*}$, with the same SER as that of $\mathcal{S}$, can be constructed in $\mathbb{R}^{N^{*}}$ via the orthonormal transformation $\mathbf{U}^{T} \mathbf{S}$ and discarding the last $N-N^{*}$ rows of zeros. Here, $\mathbf{S}=\mathbf{U} \boldsymbol{\Sigma} \mathbf{V}^{T}$ is a singular value decomposition of $\mathbf{S}$, where $\mathbf{U}$ is an $N$-by- $N$ real orthonormal matrix, $\mathbf{V}$ is an $M$-by- $M$ real orthonormal matrix, and $\Sigma$ is an $N$-by- $M$ rectangular diagonal matrix containing the singular values of $\mathbf{S}$ in descending order along its diagonal. Therefore, in the following, without loss of generality, it is assumed that the signal constellation $\mathcal{S}$ is irreducible, i.e., $\operatorname{rank}(\mathbf{S})=N$.

\section{MAIN RESULTS}

In what follows, a number of properties of the SER of the minimum distance detector is derived for arbitrary constellations under AWGN. 


\section{A. Representation of the SER}

The subsequent analysis relies on a slightly modified version of the representation for the SER that was originally established in the proof of Theorem 2 in [5] (and also employed in the proof of Theorem 1 in [7]). For any constellation of dimensionality $N \geq 2$, the conditional SER $P_{e, i}(\rho)$, given that $\mathbf{s}=\mathbf{s}_{i}$ is transmitted, can be expressed equivalently to (6) as

$$
P_{e, i}(\rho)=\int_{D \boldsymbol{\theta}} f_{\boldsymbol{\theta}}(\boldsymbol{\theta}) \int_{\frac{\rho R_{i}^{2}(\boldsymbol{\theta})}{2}}^{\infty} \frac{e^{-t} t^{N / 2-1}}{\Gamma\left(\frac{N}{2}\right)} \mathrm{d} t \mathrm{~d} \boldsymbol{\theta},
$$

where $\boldsymbol{\theta}:=\left[\theta_{1}, \ldots, \theta_{N-1}\right]$ represents the vector of angles, $R_{i}(\boldsymbol{\theta})$ is the distance between the origin and the boundary of the decision region $\Omega_{i}$ at the direction $\boldsymbol{\theta}, D \boldsymbol{\theta}$ denotes the range of the angles

$$
0 \leq \theta_{l} \leq \pi, l=1, \ldots, N-2,0 \leq \theta_{N-1} \leq 2 \pi,
$$

and $f_{\boldsymbol{\theta}}(\boldsymbol{\theta})$ is a PDF defined over the range $D \boldsymbol{\theta}$ as

$$
f_{\boldsymbol{\theta}}(\boldsymbol{\theta}):=\frac{\Gamma\left(\frac{N}{2}\right)}{2 \pi^{\frac{N}{2}}} \sin ^{N-2} \theta_{N-2} \sin ^{N-3} \theta_{N-3} \cdots \sin \theta_{1},
$$

where $\Gamma(z):=\int_{0}^{\infty} e^{-t} t^{z-1} \mathrm{~d} t$ is the Gamma function [9]. This representation is obtained by first expressing (6) in hyperspherical coordinates $(r, \boldsymbol{\theta})$, where $r:=|\mathbf{x}|$ and $\boldsymbol{\theta}$ is the vector of angles, and then substituting $t=\rho r^{2} / 2$ in the resulting integral [5], [10].

Likewise, the probability of correct decision, given that $\mathbf{s}=$ $\mathbf{s}_{i}$ is transmitted, can be represented as

$$
P_{c, i}(\rho)=\int_{D \boldsymbol{\theta}} f_{\boldsymbol{\theta}}(\boldsymbol{\theta}) \int_{0}^{\frac{\rho R_{i}^{2}(\boldsymbol{\theta})}{2}} \frac{e^{-t} t^{N / 2-1}}{\Gamma\left(\frac{N}{2}\right)} \mathrm{d} t \mathrm{~d} \boldsymbol{\theta} .
$$

It should be noted that the representations given in (8) and (11) can be extended to one-dimensional constellations (i.e., $N=1$ ) by defining $f_{\theta}(\theta):=0.5(\delta(\theta)+\delta(\theta-\pi))$, where $\delta(\cdot)$ is the Dirac delta function, and $0 \leq \theta<2 \pi$.

\section{B. Universal Bounds on the SER}

Based on this representation, the following upper and lower bounds are obtained.

Proposition 1: For any constellation of dimensionality $N \geq 1$, the SER $P_{e}$ of the minimum distance detector under AWGN is bounded as:

$$
\frac{\Gamma\left(\frac{N}{2}, \frac{d_{m a x}^{2}}{2 \sigma_{0}^{2}}\right)}{\Gamma\left(\frac{N}{2}\right)} \leq P_{e} \leq \frac{\Gamma\left(\frac{N}{2}, \frac{d_{m i n}^{2}}{2 \sigma_{0}^{2}}\right)}{\Gamma\left(\frac{N}{2}\right)} .
$$

where $\Gamma(s, x):=\int_{x}^{\infty} e^{-t} t^{s-1} \mathrm{~d} t$ is the upper incomplete Gamma function [9].

Proof: Using the definition of the upper incomplete Gamma function and substituting $\sigma_{0}^{2}=1 / \rho,(8)$ can be expressed as

$$
P_{e, i}=\mathbb{E}\left\{\frac{\Gamma\left(N / 2, R_{i}^{2}(\boldsymbol{\theta}) /\left(2 \sigma_{0}^{2}\right)\right)}{\Gamma\left(\frac{N}{2}\right)}\right\},
$$

where $\mathbb{E}\{\cdot\}$ denotes the expectation over the distribution of $\boldsymbol{\theta}$, i.e., $f_{\boldsymbol{\theta}}(\boldsymbol{\theta})$. Noting that $d_{\text {min }} \leq R_{i}(\boldsymbol{\theta}) \leq d_{\text {max }}$ for all $i$ and $\boldsymbol{\theta}$ in conjunction with the fact that $\Gamma(s, x)$ is a monotonically decreasing function of $x$, the result for the SER $P_{e}$ follows.

It should be noted that if $d_{\max }=\infty$, the lower bound in Proposition 1 is equal to zero. Furthermore, the bounds given in (12) cannot be improved without additional assumptions on the constellation geometry. To see this, suppose that all the decision regions $\Omega_{i}$ are spheres of the same radius $r$ so that $d_{m i n, i}=d_{\max , i}=r \forall i$. In this case, the lower and upper bounds are equal in (12), which implies that $P_{e}=\Gamma\left(N / 2, r^{2} /\left(2 \sigma_{0}^{2}\right)\right) / \Gamma(N / 2) .^{2}$

Next, we establish the relation between the sphere hardening argument of the channel coding theorem and the bounds given in (12). ${ }^{3}$ To that aim, we consider the codewords as symbols of an extended multidimensional constellation. According to the sphere hardening argument [11], the received signal lies on the surface of a hypersphere of radius $\sqrt{N \sigma_{0}^{2}}$ centered at the transmitted symbol with high probability (approaching 1 as $N \rightarrow \infty)$. This argument follows in a straightforward manner from Proposition 1 as explained next.

Corollary 1.1: Let $\epsilon>0$ be a fixed arbitrarily small number. For sufficiently large $N, P_{e} \approx 0$ for any code that satisfies $d_{\text {min }}^{2} \geq N\left(\sigma_{0}^{2}+\epsilon\right)$, while $P_{e} \approx 1$ for any code that satisfies $d_{\max }^{2} \leq N\left(\sigma_{0}^{2}-\epsilon\right)$ under AWGN.

Proof: See Appendix A.

The above corollary also applies to the conditional SER $P_{e, i}$ by substituting $d_{\min (\max )} \rightarrow d_{\min , i(\max , i)}$. An equivalent statement can be obtained for the conditional probability of the correct decision by recalling that $P_{c, i}=1-P_{e, i}$. The definition of $P_{c, i}$ given in (5) in conjunction with the above result implies that the received signals are concentrated within a shell of radius $\sqrt{N\left(\sigma_{0}^{2}-\epsilon\right)} \leq r \leq \sqrt{N\left(\sigma_{0}^{2}+\epsilon\right)}$ centered at the $i$ th symbol with high probability.

In what follows, various properties of the SER derivatives in SNR are explored based on the representation given in (8).

\section{Derivatives of the SER in SNR}

In this part, sufficient conditions are provided to determine the signs of the SER derivatives of all orders for arbitrary constellations under AWGN. For that purpose, the following identity is utilized. Let $h(t):=e^{-t} t^{N / 2-1}$ for $t \geq 0$ and $N \in \mathbb{N}_{>0}$. The $k$ th derivative of $h(t)$, denoted as $h^{(k)}(t)$, can be expressed using Leibniz's formula as follows [9]:

$$
h^{(k)}(t)=e^{-t} t^{N / 2-1-k} p_{k}(t),
$$

where $p_{k}(t)$ is the $k$ th degree polynomial given by

$$
p_{k}(t):=\sum_{i=0}^{k}\left(\begin{array}{l}
k \\
i
\end{array}\right) \mathbb{P}(N / 2-1, i)(-t)^{k-i},
$$

where $\mathbb{P}(z, i):=\prod_{m=0}^{i-1}(z-m)$ for $i \in \mathbb{N}_{>0}$ and $\mathbb{P}(z, 0):=1$ for all $z \in \mathbb{R}$.

Using (8) and (14), we present a generic representation for the $k$ th derivative of the conditional SER given that $\mathbf{s}=\mathbf{s}_{i}$ is transmitted. As suggested at the end of Section III-A, the definition of $f_{\boldsymbol{\theta}}(\boldsymbol{\theta})$ is extended to include one-dimensional constellations (i.e., $N=1$ ).

\footnotetext{
${ }^{2}$ For more on the spherical decision region, see [4, Sec. IV] and references therein.

${ }^{3}$ The relation between the convexity of error rates and the sphere hardening condition of the channel coding theorem was discussed in [5, Sec. IV].
} 
Lemma 1: Consider the minimum distance detector operating in the AWGN channel. For any constellation of dimensionality $N \geq 1$, the $k$ th derivative of the conditional SER $P_{e, i}^{(k)}(\rho)$ can be represented as

$$
P_{e, i}^{(k)}(\rho)=-\rho^{-k} \int_{D \boldsymbol{\theta}} g_{k}\left(\frac{\rho R_{i}^{2}(\boldsymbol{\theta})}{2}\right) f_{\boldsymbol{\theta}}(\boldsymbol{\theta}) \mathrm{d} \boldsymbol{\theta},
$$

where

$$
g_{k}(t):=\frac{e^{-t} t^{\frac{N}{2}}}{\Gamma\left(\frac{N}{2}\right)} p_{k-1}(t), \forall t \geq 0
$$

and $p_{k-1}(t)$ is as defined in (15).

Proof: The result follows by taking the $k$ th derivative of $P_{e, i}^{(k)}(\rho)$ in (8), employing the identity in (14) and rearranging the terms in the integrand.

It should be pointed out that different definitions of $g_{k}(t)$ result in different representations. However, the particular choice given in (17) will prove advantageous while determining the signs of the SER derivatives and obtaining bounds for them. It should be noted that $P_{e, i}^{(k)}(\rho)<0$ if $g_{k}\left(\rho R_{i}^{2}(\boldsymbol{\theta}) / 2\right)>0 \forall \boldsymbol{\theta} \in D \boldsymbol{\theta}$ since $f_{\boldsymbol{\theta}}(\boldsymbol{\theta})$ is a PDF. Similarly, $P_{e, i}^{(k)}(\rho)>0$ if $g_{k}\left(\rho R_{i}^{2}(\boldsymbol{\theta}) / 2\right)<0 \forall \boldsymbol{\theta} \in D \boldsymbol{\theta}$. From (17), it is seen that the sign of $g_{k}(t)$ is determined by the sign of the polynomial $p_{k-1}(t)$.

The following proposition provides a general procedure to determine the signs of the SER derivatives of all orders for arbitrary constellations under AWGN. A similar result for the conditional SER $P_{e, i}$, given that $\mathbf{s}=\mathbf{s}_{i}$ is transmitted, can be obtained by substituting $d_{\min (\max )} \rightarrow d_{\min , i(\max , i)}$.

Proposition 2: Consider an arbitrary constellation of dimensionality $N \geq 1$ impaired by AWGN. Let the polynomial $p_{k-1}(t)$ be defined as in (15) for some $k \geq 1$. Let $r$ denote the number of positive real roots of $p_{k-1}(t)$ with odd multiplicity 4 that are arranged in ascending order, i.e., $t_{1}<t_{2}<\cdots<t_{r}$, where $t_{i}$ denotes the $i$ th such root. The $k$ th derivative of the SER $P_{e}^{(k)}(\rho)$ in SNR satisfies the following properties:

1) If $r=0,(-1)^{k} P_{e}^{(k)}(\rho)>0$ for all $\rho>0$.

2) If $r \geq 1$,

(2a) At low SNR region specified by $\rho<2 t_{1} / d_{\text {max }}^{2}$,

$$
\left\{\begin{array}{ll}
P_{e}^{(k)}(\rho)<0, & \text { if } k<N / 2+1 \\
(-1)^{k-\left\lfloor\frac{N-1}{2}\right\rfloor} P_{e}^{(k)}(\rho)>0, & \text { if } k \geq N / 2+1
\end{array},\right.
$$

(2b) At intermediate SNR regions specified by $2 t_{m} / d_{\min }^{2}<\rho<2 t_{m+1} / d_{\text {max }}^{2}$ for $m \in$ $\{1,2, \ldots, r-1\},(-1)^{k+r-m} P_{e}^{(k)}(\rho)>0$,

(2c) At high SNR region specified by $\rho>2 t_{r} / d_{\text {min }}^{2}$, $(-1)^{k} P_{e}^{(k)}(\rho)>0$.

Proof: See Appendix B.

Although the convexity properties of the SER were studied in [4], [5] for arbitrary constellations (i.e., $N \geq 1$ ), and the complete monotonicity of the SER was established in [6] for oneand two-dimensional constellations (i.e., $N \in\{1,2\}$ ), the results presented in this paper cover those in [4]-[6] as special cases, and extend them to higher order derivatives of the SER. It should be noted that such an analysis was avoided in the referenced papers as it was considered to be rather involved. In what

${ }^{4}$ The polynomial $p_{k-1}(t)$ changes sign at the real roots of odd multiplicity. follows, we provide three examples to corroborate the results described in Proposition 2.

Example 2.1 [5, Th. 2]: Consider the second derivative (i.e., $k=2$ ) of the SER of the minimum distance detector for arbitrary constellations in $\mathbb{R}^{N}$ impaired by AWGN. From (15), it is seen that $p_{1}(t)=-t+N / 2-1$ with a single root at $t=N / 2-1$. The convexity of the SER for arbitrary one- and two-dimensional constellations can be verified from Proposition 2 by noting that there is no positive root of $p_{1}(t)$ for $N \in\{1,2\}$. Next, we consider the case for $N \geq 3$. Since $p_{1}(t)>0$ for $t \in(0, N / 2-1)$, it is concluded that $P_{e}^{(2)}(\rho)<0$ for all $\rho<$ $(N-2) / d_{\text {max }}^{2}$. Similarly, since $p_{1}(t)<0$ for $t \in(N / 2-1, \infty)$, we get $P_{e}^{(2)}(\rho)>0$ for all $\rho>(N-2) / d_{\text {min }}^{2}$. Again, these results can be obtained from Proposition 2 by substituting $r=1$, $k=2, t_{1}=N / 2-1$ and $N \geq 3$.

Example 2.2 [6, Th. 1]: For arbitrary one- and two-dimensional constellations, the SER is c.m. in SNR, i.e., the sign of the $k$ th derivative of the SER in SNR satisfies:

$$
(-1)^{k} \frac{d^{k}}{d \rho^{k}} P_{e}(\rho) \geq 0, \quad \forall \rho>0
$$

for all $k \in \mathbb{N}_{\geq 0}$ under $\mathrm{AWGN}$ and minimum distance detection. ${ }^{5}$ This result can be established using Proposition 2 as shown next. The case for $k=0$ follows from the nonnegativity of the SER. For $k=1$, since $p_{0}(t)=1$, there is no positive root and we get $P_{e}^{(1)}(\rho)<0$ for all $\rho>0$ and $N \geq 1$ from Proposition 2 . For $k \geq 2$, one- and two-dimensional constellations are treated separately. First, we consider one-dimensional constellations, i.e., $N=1$. Let $p_{k-1}(t):=a_{0}+a_{1} t+\cdots+a_{k-1} t^{k-1}$. From (15), it is seen that the coefficient multiplying the term $t^{i}$ is given by $a_{i}=(-1)^{i}\left(\begin{array}{c}k-1 \\ i\end{array}\right) \mathbb{P}(-0.5, k-1-i)$ for $0 \leq i \leq k-1$, which, in conjunction with the definition of $\mathbb{P}(\cdot)$ after $(15)$, implies that all the coefficients of $p_{k-1}(t)$ have the same sign as that of $(-1)^{k-1}$. Consequently, there is no positive root of $p_{k-1}(t)$, and we have $(-1)^{k} P_{e}^{(k)}(\rho)>0$ for all $\rho>0$ by substituting $r=0$ in Proposition 2. Next, we consider two-dimensional constellations, i.e., $N=2$. Since $p_{k-1}(t)=(-t)^{k-1}$ for $N=2$, there is no positive root of $p_{k-1}(t)$ in this case as well and the same conclusion follows.

Example 2.3: Consider the third derivative (i.e., $k=3$ ) of the SER of the minimum distance detector for arbitrary constellations in $\mathbb{R}^{N}$ impaired by AWGN. From (15), we get $p_{2}(t)=$ $t^{2}-(N-2) t+(N / 2-1)(N / 2-2)$ with roots at $(N / 2-$ $1)-\sqrt{N / 2-1}$ and $(N / 2-1)+\sqrt{N / 2-1}$. For $N \in\{1,2\}$, $p_{2}(t)>0$ for all $t>0$, which implies that $P_{e}^{(3)}(\rho)<0$ for all $\rho>0$. This can also be seen from Proposition 2 since there is no positive root for $N \in\{1,2\}$. For $N=3$ and $N=4$, $t_{1}:=(N / 2-1)+\sqrt{N / 2-1}$ is the only positive root, and we get $p_{2}(t)<0$ for $t \in\left(0, t_{1}\right)$, and $p_{2}(t)>0$ for $t>t_{1}$. Then, it is concluded that for $N=3, P_{e}^{(3)}(\rho)>0$ for all $\rho<$ $(1+\sqrt{2}) / d_{\text {max }}^{2}$, and $P_{e}^{(3)}(\rho)<0$ for all $\rho>(1+\sqrt{2}) / d_{\text {min }}^{2}$. Similarly, for $N=4, P_{e}^{(3)}(\rho)>0$ for all $\rho<4 / d_{\max }^{2}$, and $P_{e}^{(3)}(\rho)<0$ for all $\rho>4 / d_{\text {min }}^{2}$. These results can be obtained from Proposition 2 by substituting $r=1, k=3$, $t_{1}=(N / 2-1)+\sqrt{N / 2-1}$ and $N \in\{3,4\}$. Next, we consider the case for $N \geq 5$. There are two positive roots at $t_{1}:=$ $(N / 2-1)-\sqrt{N / 2-1}$ and $t_{2}:=(N / 2-1)+\sqrt{N / 2-1}$,

\footnotetext{
${ }^{5}$ The derivative of order $k=0$ is defined as $P_{e}(\rho)$ itself.
} 
which imply that $p_{2}(t)>0$ for $t \in\left(0, t_{1}\right)$ and $t>t_{2}$, while $p_{2}(t)<0$ for $t \in\left(t_{1}, t_{2}\right)$. Consequently, $P_{e}^{(3)}(\rho)<0$ for all $\rho<2 t_{1} / d_{\text {max }}^{2}$ and $\rho>2 t_{2} / d_{\text {min }}^{2}$, whereas $P_{e}^{(3)}(\rho)>0$ for $2 t_{1} / d_{\min }^{2}<\rho<2 t_{2} / d_{\max }^{2}$. Again, these results can be obtained from Proposition 2 by substituting $r=2, k=3$, $t_{1}=(N / 2-1)-\sqrt{N / 2-1}, t_{2}=(N / 2-1)+\sqrt{N / 2-1}$ and $N>5$.

The sufficient conditions described in Proposition 2 help determine the sign of the SER derivatives of all orders. We remark that for higher order derivatives, the roots of the polynomial $p_{k-1}(t)$ needs to be computed numerically, for which more and more efficient algorithms are being developed [12]. Alternatively, the following corollary can be applied to determine the high SNR region where the condition $(-1)^{k} P_{e}^{(k)}(\rho)>0$ given in item (2c) of Proposition 2 is satisfied. A similar corollary can be obtained for the derivatives of the conditional SER $P_{e, i}^{(k)}(\rho)$ by substituting $d_{\min } \rightarrow d_{\min , i}$ in the following.

Corollary 2.1: For arbitrary constellations impaired by AWGN, the $k$ th derivative of the SER of the minimum distance detector $P_{e}^{(k)}(\rho)$ satisfies $(-1)^{k} P_{e}^{(k)}(\rho)>0$ when $\rho \geq 2 \alpha / d_{\text {min }}^{2}$, where $\alpha$ is given by

$\alpha:=2 \max \left\{\left|\frac{a_{k-2}}{a_{k-1}}\right|,\left|\frac{a_{k-3}}{a_{k-1}}\right|^{\frac{1}{2}}, \ldots,\left|\frac{a_{1}}{a_{k-1}}\right|^{\frac{1}{k-2}},\left|\frac{a_{0}}{2 a_{k-1}}\right|^{\frac{1}{k-1}}\right\}$

and $a_{i}:=(-1)^{i}\left(\begin{array}{c}k-1 \\ i\end{array}\right) \mathbb{P}(N / 2-1, k-1-i)$ for $i=0,1, \ldots, k-$ 1 , and $\mathbb{P}($.$) is as defined on the line following (15). A simpler$ but larger choice for $\alpha$ is $\alpha:=(k-1)(N+2)$.

Proof: See Appendix C.

\section{Universal Bounds on the Derivatives of the SER}

In this section, generic lower and upper bounds are obtained for the SER derivatives of all orders based on the representation given in (16). These bounds depend only on SNR, constellation dimensionality, and order of the derivative. Our analysis generalizes the results obtained in [4, Sec. IV] to higher order derivatives.

Proposition 3: For arbitrary constellations impaired by AWGN, the $k$ th derivative of the SER in SNR $P_{e}^{(k)}(\rho)$ (and also $\left.P_{e, i}^{(k)}(\rho)\right)$ is bounded, under minimum distance detection, as follows:

$$
\frac{c_{l}}{\rho^{k}} \leq P_{e}^{(k)}(\rho) \leq \frac{c_{u}}{\rho^{k}},
$$

where $c_{l}$ and $c_{u}$ are defined as

$$
\begin{gathered}
c_{l}:=-\max _{t \in \mathcal{C}_{+} \cup\{0\}} \frac{e^{-t} t^{\frac{N}{2}}}{\Gamma\left(\frac{N}{2}\right)} p_{k-1}(t), \\
c_{u}:=-\min _{t \in \mathcal{C}_{+} \cup\{0\}} \frac{e^{-t} t^{\frac{N}{2}}}{\Gamma\left(\frac{N}{2}\right)} p_{k-1}(t),
\end{gathered}
$$

and $\mathcal{C}_{+}$denotes the set of the positive real roots of the polynomial $z(t):=p_{k}(t)+k p_{k-1}(t)$ with odd multiplicity, where $p_{k}(t)$ is as defined in (15).

Proof: See Appendix D.

It should be noted that the magnitude of $k$ th SER derivative $\left|P_{e}^{(k)}(\rho)\right|$ decreases at least as $1 / \rho^{k}$ up to a multiplicative constant. Based on Proposition 3, the following results can be obtained in a straightforward manner. For completeness, we start with the bound on the first derivative which was originally stated in [4, Th. 6], and provide an alternative proof.

Example 3.1 [4, Th. 6]: For arbitrary constellations impaired by AWGN, the first derivative of the SER in SNR $P_{e}^{(1)}(\rho)$ (and also $\left.P_{e, i}^{(1)}(\rho)\right)$ is bounded, under minimum distance detection, as follows:

$$
-\frac{e^{-N / 2}\left(\frac{N}{2}\right)^{\frac{N}{2}}}{\Gamma\left(\frac{N}{2}\right) \rho} \leq P_{e}^{(1)}(\rho) \leq 0,
$$

and the lower bound is achieved for spherical decision region, $\Omega_{i}=\left\{\mathbf{x}:|\mathbf{x}|^{2} \leq N / \rho\right\}$. This result can be established using Proposition 3 as shown next. For $k=1, z(t)=-t+N / 2$, which has a single positive root at $N / 2$. Substituting $\mathcal{C}_{+}=$ $\{N / 2\}$ into (21) and (22), and recalling that $p_{0}(t)=1$, we get $c_{l}=-\frac{e^{-N / 2}(N / 2)^{N / 2}}{\Gamma(N / 2)}$ and $c_{u}=0$. Furthermore, since the maximum of $g_{k}(t)$ is attained at $t=N / 2$, it is seen from (16) that if $R_{i}^{2}(\boldsymbol{\theta})=N / \rho$ for all $\boldsymbol{\theta}$, the lower bound is achieved.

The next example corrects the bounds given in [4, Th. 7] for the second derivative of the SER.

Example 3.2 [4, Th. 7]: For arbitrary constellations impaired by AWGN, the second derivative of the SER in SNR $P_{e}^{(2)}(\rho)$ (and also $P_{e, i}^{(2)}(\rho)$ ) is bounded, under minimum distance detection, as follows:

$$
\begin{array}{r}
-\frac{e^{-\left(\frac{N}{2}-\sqrt{\frac{N}{2}}\right)}\left(\frac{N}{2}-\sqrt{\frac{N}{2}}\right)^{\frac{N}{2}}\left(\sqrt{\frac{N}{2}}-1\right)^{+}}{\Gamma\left(\frac{N}{2}\right) \rho^{2}} \leq P_{e}^{(2)}(\rho) \\
\leq \frac{e^{-\left(\frac{N}{2}+\sqrt{\frac{N}{2}}\right)}\left(\frac{N}{2}+\sqrt{\frac{N}{2}}\right)^{\frac{N}{2}}\left(1+\sqrt{\frac{N}{2}}\right)}{\Gamma\left(\frac{N}{2}\right) \rho^{2}},
\end{array}
$$

where $(x)^{+}=x$ if $x \geq 0$ and 0 otherwise. The upper bound is achieved for the spherical decision region, $\Omega_{i}=\left\{\mathbf{x}:|\mathbf{x}|^{2} \leq\right.$ $(N+\sqrt{2 N}) / \rho\}$ for all $N \geq 1$. The lower bound is achieved for the spherical decision region, $\Omega_{i}=\left\{\mathbf{x}:|\mathbf{x}|^{2} \leq(N-\sqrt{2 N}) / \rho\right\}$ for $N \geq 3$. This result can be established using Proposition 3 as follows. For $k=2, z(t)=t^{2}-N t+N(N-2) / 4$ with roots at $N / 2-\sqrt{N / 2}$ and $N / 2+\sqrt{N / 2}$. For $N \in\{1,2\}$, there is a single positive root at $N / 2+\sqrt{N / 2}$. Substituting into (21) and (22), we get $c_{l}=0$ and $c_{u}=e^{-(N / 2+\sqrt{N / 2})}(N / 2+$ $\sqrt{N / 2})^{N / 2}(1+\sqrt{N / 2}) / \Gamma(N / 2)$. In the case of $N \geq 3$, both roots are positive, and we get $c_{l}=-e^{-(N / 2-\sqrt{N / 2})}(N / 2-$ $\sqrt{N / 2})^{N / 2}(\sqrt{N / 2}-1) / \Gamma(N / 2)$ and $c_{u}$ remains the same. It can be seen from (16) that the nonzero lower and upper bounds are achieved if $R_{i}^{2}(\boldsymbol{\theta})=(N-\sqrt{2 N}) / \rho$ and $R_{i}^{2}(\boldsymbol{\theta})=(N+$ $\sqrt{2 N}) / \rho$ for all $\boldsymbol{\theta}$, respectively.

The following corollary provides bounds on the $k$ th derivative of the SER for arbitrary two-dimensional constellations, which are stronger that the complete monotonicity stated in $[6$, Th. 1].

Corollary 3.1: For arbitrary two-dimensional constellations impaired by AWGN, the $k$ th derivative of the SER in SNR $P_{e}^{(k)}(\rho)$ (and also $P_{e, i}^{(k)}(\rho)$ ) is bounded, under minimum distance detection, as follows:

$$
0 \leq(-1)^{k} P_{e}^{(k)}(\rho) \leq \frac{e^{-k} k^{k}}{\rho^{k}}
$$


The nonzero bound is achieved for the spherical decision region, $\Omega_{i}=\left\{\mathbf{x}:|\mathbf{x}|^{2} \leq 2 k / \rho\right\}$ for all $k \geq 1$.

Proof: For $N=2$, it is recalled from (15) that $p_{k}(t)=$ $(-t)^{k}$. Consequently, $z(t)=(-t)^{k-1}(-t+k)$ with a single positive root at $k$. Substituting into (21) and (22), we get $c_{l}=$ $-e^{-k} k^{k}$ and $c_{u}=0$ for odd $k$, while $c_{l}=0$ and $c_{u}=e^{-k} k^{k}$ for even $k$. Furthermore, since the maximum (minimum) of $g_{k}(t)$ is attained at $t=k$ for odd (even) $k$, if $R_{i}^{2}(\boldsymbol{\theta})=2 k / \rho$ is satisfied for all $\boldsymbol{\theta}$, the lower (upper) bound is achieved.

As expected, the results of Corollary 3.1 for $k=1$ and $k=2$ agree with those of Example 3.1 and Example 3.2 when $N=2$ is substituted into (23) and (24), respectively.

Corollary 3.2: The asymptotic behavior of the SER derivatives, which is also valid for the conditional SER derivatives, is characterized as

$$
\lim _{\rho \rightarrow \infty} P_{e}^{(k)}(\rho)=0 \quad \forall k,
$$

and the convergence to the limit is uniform.

Proof: Immediate from Proposition 3.

This result can also be justified by the fact that $(-1)^{k} P_{e}^{(k)}(\rho)>0$ at high SNR. Since the $(k-1)$ th derivative has the opposite sign of the $k$ th derivative, the $k$ th derivative has to approach zero to avoid a sign change for the $(k-1)$ th derivative.

The bounds proposed in this section can be used in the design and analysis of optimization algorithms: e.g., to analyze the convergence conditions and rates, and to develop stopping criteria based on the suboptimality of the solutions [4], [8, Ch. 9-11]. Evidently, the bounds on the first and second derivatives are the ones that could be employed most commonly in an optimization problem, e.g., while determining the required step size of gradient methods. The assignment of a small value would cause the algorithm to converge slowly, whereas assigning too high a value could result in missing an optimal point. On the other hand, the bounds on the higher order derivatives can be equally important. For example, the Lipschitz coefficient of a function $f$ can be interpreted as a bound on the third derivative of $f$, and measures how well $f$ can be approximated by a quadratic model [8, Sec. 9.5.3]. As a result, it plays a prominent role in the performance of Newton's method, which is expected to yield good performance for a function whose quadratic model varies slowly. Being a bound on the third derivative, the Lipschitz coefficient for the error probability function can be determined using the framework developed in this section. By a similar reasoning, the bounds on higher order derivatives of the SER can prove useful when higher order models are employed to approximate the error probability. Moreover, the constellation-independent nature of the proposed bounds makes them a valuable tool in the design of optimization algorithms that are required to work in a variety of communication settings.

\section{CONCLUding REMARKS}

In this paper, the spherical symmetry of the Gaussian noise distribution was exploited to obtain universal bounds on the SER and its higher order derivatives for arbitrary constellations impaired by AWGN, when the minimum distance detector is used. Using the derived bounds on the SER, we provided an alternative proof of the sphere hardening argument. We also presented a general procedure that tests for sufficient conditions to determine the sign of the SER derivative of any order. This method is based on the positive real roots (with odd multiplicity) of an explicitly-specified polynomial, which can be computed efficiently using standard numerical algorithms [12]. Nevertheless, simpler bounds were provided to determine the high SNR region, where the sign of the $k$ th derivative is given by that of $(-1)^{k}$, in order to alleviate the need for the computation of the roots. Universal bounds were proposed for SER derivatives of all orders. These bounds revealed a stronger characterization of the SER for arbitrary two-dimensional constellations, which had been shown to be completely monotone in a relevant work.

It can be seen that the results presented in this paper are also valid in the correlated noise scenario, since a whitening filter can be applied to the received signal and an equivalent constellation with identical performance can be obtained. Likewise, the extension to interference channel (as in multiuser communications) is straightforward when the interference can be modeled as Gaussian noise [13]. The bounds and the properties of the SER derivatives derived in this paper also apply to the bit error rate (BER) when the latter can be expressed as a nonnegative sum of conditional SERs or approximated as $\mathrm{BER} \approx \mathrm{SER} / \log _{2} M$ [2], [14]. As pointed out in [4], [5], the reported results can be applied to coded systems by considering codewords as symbols of an extended multidimensional constellation. Finally, we note that a generic detector with centerconvex decision regions, as defined in [5, Sec. III-A], can be employed at the receiver instead of the minimum distance detector, without altering the results.

\section{APPENDIX A \\ PROOF OF COROLlaRY 1.1}

For any code whose decision regions enclose the spheres of radius $\sqrt{N \sigma_{0}^{2}}$, i.e., $d_{\min }^{2} \geq N\left(\sigma_{0}^{2}+\epsilon\right)$, the following holds due to monotonicity of the upper incomplete Gamma function given in (12):

$$
P_{e} \leq \frac{\Gamma\left(N / 2, N / 2\left(1+\epsilon^{\prime}\right)\right)}{\Gamma\left(\frac{N}{2}\right)},
$$

where $\epsilon^{\prime}:=\epsilon / \sigma_{0}^{2}$. On the other hand, for any code whose decision regions are enclosed by the spheres of radius $\sqrt{N \sigma_{0}^{2}}$, i.e., $d_{\max }^{2} \leq N\left(\sigma_{0}^{2}-\epsilon\right)$, we get from (12):

$$
P_{e} \geq \frac{\Gamma\left(N / 2, N / 2\left(1-\epsilon^{\prime}\right)\right)}{\Gamma\left(\frac{N}{2}\right)} .
$$

For the above two cases, the aim is to determine the behavior of the SER $P_{e}$ as $N \rightarrow \infty$. To that aim, it is recalled that

$$
\frac{\Gamma\left(N / 2, N / 2\left(1+\epsilon^{\prime}\right)\right)}{\Gamma\left(\frac{N}{2}\right)}=\int_{N / 2\left(1+\epsilon^{\prime}\right)}^{\infty} \frac{e^{-t} t^{N / 2-1}}{\Gamma\left(\frac{N}{2}\right)} \mathrm{d} t .
$$

Notice that the integrand in (29) is the Gamma PDF with shape parameter $N / 2$ and scale parameter 1 , which is denoted by $\operatorname{Gamma}(N / 2,1)$ [15]. Let $X_{1}, X_{2}, \ldots, X_{N}$ be independent and identically distributed (i.i.d.) Gamma $(1 / 2,1)$ RVs. It is well known that sum of i.i.d. Gamma RVs is also Gamma distributed, i.e., $\sum_{i=1}^{N} X_{i} \sim \operatorname{Gamma}(N / 2,1)$ [15]. Then, the integral given in (29) can be expressed in terms of the 
complementary cumulative distribution function (CCDF) of $\sum_{i=1}^{N} X_{i}$, that is,

$$
\frac{\Gamma\left(N / 2, N / 2\left(1+\epsilon^{\prime}\right)\right)}{\Gamma\left(\frac{N}{2}\right)}=\operatorname{Pr}\left[\sum_{i=1}^{N} X_{i} \geq \frac{N}{2}\left(1+\epsilon^{\prime}\right)\right]
$$

By letting $N \rightarrow \infty$,

$$
\begin{aligned}
& \lim _{N \rightarrow \infty} \frac{\Gamma\left(N / 2, N / 2\left(1+\epsilon^{\prime}\right)\right)}{\Gamma\left(\frac{N}{2}\right)} \\
& \quad=\lim _{N \rightarrow \infty} \operatorname{Pr}\left[\frac{1}{N} \sum_{i=1}^{N} X_{i}-\frac{1}{2} \geq \frac{\epsilon^{\prime}}{2}\right] \\
& \leq \lim _{N \rightarrow \infty} \operatorname{Pr}\left[\left|\frac{1}{N} \sum_{i=1}^{N} X_{i}-\frac{1}{2}\right| \geq \frac{\epsilon^{\prime}}{2}\right] \\
& \quad=0,
\end{aligned}
$$

where the last line follows from the weak law of large numbers [15].

Similarly,

$$
\begin{aligned}
& \lim _{N \rightarrow \infty} \frac{\Gamma\left(N / 2, N / 2\left(1-\epsilon^{\prime}\right)\right)}{\Gamma\left(\frac{N}{2}\right)} \\
& =\lim _{N \rightarrow \infty} \operatorname{Pr}\left[\sum_{i=1}^{N} X_{i} \geq \frac{N}{2}\left(1-\epsilon^{\prime}\right)\right] \\
& =\lim _{N \rightarrow \infty} \operatorname{Pr}\left[\frac{1}{N} \sum_{i=1}^{N} X_{i}-\frac{1}{2} \geq-\frac{\epsilon^{\prime}}{2}\right] \\
& \geq \lim _{N \rightarrow \infty} \operatorname{Pr}\left[\left|\frac{1}{N} \sum_{i=1}^{N} X_{i}-\frac{1}{2}\right| \leq \frac{\epsilon^{\prime}}{2}\right] \\
& =1 .
\end{aligned}
$$

Inequalities (31) and (32) in conjunction with the bounds given in (27) and (28) establish the result.

\section{APPENDIX B \\ ProOF OF PROPOSITION 2}

Recalling that $p_{k-1}(t)$ is a polynomial of degree $k-1$, it has $k-1$ roots [16]. These roots can be obtained in closed form for polynomials of degree less than five, while efficient numerical algorithms exist for roots of higher-degree polynomials [12]. In order to derive sufficient conditions to determine the signs of the SER derivatives, we will concentrate only on the positive real roots, where $p_{k-1}(t)$ changes sign. First, we consider the case when there is no positive real root for a given $p_{k-1}(t)$. Then, the sign of $P_{e, i}^{(k)}(\rho)$ is the opposite of the sign of $p_{k-1}(t)$ over the interval $t \in(0, \infty)$, which can be obtained from the sign of the term with the highest exponent in $p_{k-1}(t)$, i.e., $(-1)^{k-1} t^{k-1}$. Taking into account the leading negative sign in (16), it is seen that the sign of $P_{e, i}^{(k)}(\rho)$ is the same as that of $(-1)^{k}$ for all $\rho>0$.

Next, we consider the case when there exists at least one positive real root. Let $r$ denote the number of such roots $(r \leq k-1)$, which are arranged in increasing order, i.e., $t_{1}<\cdots<t_{r}$. We first consider the low SNR regime. If $\rho<2 t_{1} / R_{i}^{2}(\boldsymbol{\theta})$ for all $\boldsymbol{\theta} \in D \boldsymbol{\theta}$ (or equivalently if $\rho<2 t_{1} / d_{\max , i}^{2}$ ), then it is evident from (16) that the sign of $P_{e, i}^{(k)}(\rho)$ is the opposite of the sign of $p_{k-1}(t)$ over the interval $t \in\left(0, t_{1}\right)$, which is determined by the sign of the term with the lowest exponent in $p_{k-1}(t) \mathrm{de}$ fined in (15). For $k<N / 2+1$, this is the constant term of the polynomial, i.e., $\mathbb{P}(N / 2-1, k-1)$, which is positive. For $k \geq N / 2+1$ with odd $N$, the sign of $\mathbb{P}(N / 2-1, k-1)$ is the same as that of $(-1)^{k-\frac{N+1}{2}}$. For $k \geq N / 2+1$ with even $N$, the lowest degree term of $p_{k-1}(t)$ is $(-1)^{i}\left(\begin{array}{c}k-1 \\ i\end{array}\right)(N / 2-1) ! t^{i}$ where $i=k-N / 2$. This is because $\mathbb{P}(N / 2-1, m)=0$ for all $m \geq N / 2$ if $N$ is an even positive integer. Hence, the sign of $p_{k-1}(t)$ over the interval $t \in\left(0, t_{1}\right)$ is the sign of $(-1)^{k-N / 2}$. Next, we consider the high SNR regime. If $\rho>2 t_{r} / R_{i}^{2}(\boldsymbol{\theta})$ for all $\boldsymbol{\theta} \in D \boldsymbol{\theta}$ (or equivalently if $\rho>2 t_{r} / d_{\min , i}^{2}$ ), then the sign of $P_{e, i}^{(k)}(\rho)$ is the opposite of the sign of $p_{k-1}(t)$ over the interval $t \in\left(t_{r}, \infty\right)$, which is governed by the sign of the term with the highest exponent in $p_{k-1}(t)$. Since this term is $(-1)^{k-1} t^{k-1}$, the sign of $P_{e, i}^{(k)}(\rho)$ is seen to be the same as that of $(-1)^{k}$ for all $\rho>2 t_{r} / d_{m i n, i}^{2}$ after accounting for the leading negative sign in (16). Furthermore, if $t_{m} / d_{m i n, i}^{2}<t_{m+1} / d_{\max , i}^{2}$ is satisfied for some $m \in\{1, \ldots, r-1\}$, then the sign of $P_{e, i}^{(k)}(\rho)$ for all $\rho \in\left(2 t_{m} / d_{m i n, i}^{2}, 2 t_{m+1} / d_{m a x, i}^{2}\right)$ is the opposite of the sign of $p_{k-1}(t)$ over the interval $t \in\left(t_{m}, t_{m+1}\right)$. This is because the sign of $p_{k-1}(t)$ remains the same over the interval $t \in\left(t_{m}, t_{m+1}\right)$.

Since the SER $P_{e}^{(k)}(\rho)$ is given as a nonnegative sum of the conditional SERs, the results in the proposition follow by substituting $d_{\min , i(\max , i)} \rightarrow d_{\min (\max )}$.

\section{APPENDIX C}

PROOF OF COROLlARY 2.1

In Proposition 2, it is stated that $(-1)^{k} P_{e}^{(k)}(\rho)>0$ for $\rho>2 t_{r} / d_{\text {min }}^{2}$, where $t_{r}$ is the largest positive real root of the polynomial $p_{k-1}(t)$. The idea is to find an upper bound on $t_{r}$ and substitute this bound for $t_{r}$ in the above condition. Let $p_{k-1}(t):=a_{0}+a_{1} t+\cdots+a_{k-1} t^{k-1}$. From (15), it is seen that the coefficient multiplying the term $t^{i}$ is given by $a_{i}=(-1)^{i}\left(\begin{array}{c}k-1 \\ i\end{array}\right) \mathbb{P}(N / 2-1, k-1-i)$ for $0 \leq i \leq k-1$. A powerful bound on the magnitude of the roots of a polynomial is given by the Fujiwara's bound [17], which is shown in (19). The second choice is derived from the Kojima's bound [18]. Although the Fujiwara's bound always compares favorably with the Kojima's bound [19], the latter leads to a simpler expression for $\alpha$. For a polynomial of degree $k-1$, Kojima's bound is given by [18]

$$
2 \max \left\{\left|\frac{a_{k-2}}{a_{k-1}}\right|,\left|\frac{a_{k-3}}{a_{k-2}}\right|, \ldots,\left|\frac{a_{1}}{a_{2}}\right|,\left|\frac{a_{0}}{2 a_{1}}\right|\right\} .
$$

Based on the representation of $p_{k-1}(t)$ given above, for $i=$ $0,1, \ldots, k-2$, we get

$$
\begin{aligned}
\left|\frac{a_{i}}{a_{i+1}}\right| & =\left|(i+1)\left(\frac{N}{2(k-i-1)}-1\right)\right|, \\
& \leq(i+1)\left(\frac{N}{2(k-i-1)}+1\right), \\
& \leq(k-1)\left(\frac{N}{2}+1\right) .
\end{aligned}
$$

Hence, an upper bound on the Kojima's bound, and therefore on $t_{r}$, is $(k-1)(N+2)$. 


\section{APPENDIX D \\ PROOF OF PROPOSITION 3}

From (16), it is noted that the conditional SER can be expressed as

$$
P_{e, i}^{(k)}(\rho)=-\rho^{-k} \mathbb{E}\left\{g_{k}\left(\frac{\rho R_{i}^{2}(\boldsymbol{\theta})}{2}\right)\right\},
$$

where the expectation is taken over the distribution of $\boldsymbol{\theta}$. From the definition of $g_{k}(t)$ given in (17), it is seen that $g_{k}(t)$ is a bounded smooth function for $t>0, g_{k}(0)=0$ and $\lim _{t \rightarrow \infty} g_{k}(t)=0$ for all $k \geq 1$. Consequently, $g_{k}\left(\rho R_{i}^{2}(\boldsymbol{\theta}) / 2\right)$ can be upper and lower bounded as

$$
\min _{t \geq 0} g_{k}(t) \leq g_{k}\left(\frac{\rho R_{i}^{2}(\boldsymbol{\theta})}{2}\right) \leq \max _{t \geq 0} g_{k}(t) .
$$

Since this holds for all $\rho \geq 0, \boldsymbol{\theta} \in D \boldsymbol{\theta}$ and $i \in\{1, \ldots, M\}$, we get

$$
-\rho^{-k} \max _{t \geq 0} g_{k}(t) \leq P_{e}^{(k)}(\rho) \leq-\rho^{-k} \min _{t \geq 0} g_{k}(t) .
$$

The global minimum and maximum of $g_{k}(t)$ over the interval $t \geq 0$ can be obtained by computing the values of $g_{k}(t)$ at the positive real roots of its derivative with odd multiplicity together with the boundary point $t=0$, and selecting the corresponding extremum. In order to obtain the derivative of $g_{k}(t)$, we express it as the product of two terms, i.e., $g_{k}(t)=$ $g_{k, 1}(t) g_{k, 2}(t)$, where $g_{k, 1}(t):=e^{-t} t^{N / 2-1-(k-1)} p_{k-1}(t)$ and $g_{k, 2}(t):=t^{k} / \Gamma(N / 2)$. From (14), it is noted that the first term $g_{k, 1}(t)$ is the $(k-1)$ th derivative of $e^{-t} t^{N / 2-1}$. By applying the rule for the derivative of a product, we get $\frac{d}{d t}\left(g_{k}(t)\right)=\frac{d}{d t}\left(g_{k, 1}(t)\right) g_{k, 2}(t)+g_{k, 1}(t) \frac{d}{d t}\left(g_{k, 2}(t)\right)$. The term $\frac{d}{d t}\left(g_{k, 1}(t)\right)$ is the $k$ th derivative of $e^{-t} t^{N / 2-1}$, which can be obtained using (14). After some manipulation, the derivative of $g_{k}(t)$ can be written as

$$
\frac{d}{d t}\left(g_{k}(t)\right)=\frac{e^{-t} t^{N / 2-1}}{\Gamma\left(\frac{N}{2}\right)}\left(p_{k}(t)+k p_{k-1}(t)\right) .
$$

Since $e^{-t} t^{N / 2-1} / \Gamma(N / 2)>0$ for $t>0$, the candidate nonzero extrema points are given by the positive real roots of $\left(p_{k}(t)+k p_{k-1}(t)\right)$ with odd multiplicity, which is denoted with $\mathcal{C}_{+}$. Since efficient algorithms exist for obtaining polynomial roots, instead of checking for all $t>0$, the extrema of $g_{k}(t)$ can be computed over the set $\mathcal{C}_{+} \cup\{0\}$. The proposition follows by substituting for corresponding expressions in (37).

\section{REFERENCES}

[1] J. M. Wozencraft and I. M. Jacobs, Principles of Communication Engineering. New York, NY, USA: Wiley, 1965.

[2] J. G. Proakis, Digital Communications, 4th ed. New York, NY, USA: McGraw-Hill, 2001.

[3] M. K. Simon and M. S. Alouini, Digital Communication Over Fading Channels. New York, NY, USA: Wiley, 2005.

[4] S. Loyka, V. Kostina, and F. Gagnon, "Error rates of the maximumlikelihood detector for arbitrary constellations: Convex/concave behavior and applications," IEEE Trans. Inform. Theory, vol. 56, no. 4, pp. 1948-1960, Apr. 2010.

[5] S. Loyka, V. Kostina, and F. Gagnon, "On convexity of error rates in digital communications," IEEE Trans. Inform. Theory, vol. 59, no. 10, pp. 6501-6516, Oct. 2013.

[6] A. Rajan and C. Tepedelenlioglu, "On the complete monotonicity of symbol error rates," in Proc. IEEE Int. Symp. Inform. Theory (ISIT), Jul. 1-6, 2012, pp. 1430-1434.

[7] A. Rajan and C. Tepedelenlioglu, "A representation for the symbol error rate using completely monotone functions," IEEE Trans. Inform. Theory, vol. 59, no. 6, pp. 3922-3931, Jun. 2013.

[8] S. Boyd and L. Vandenberghe, Convex Optimization. Cambridge, U.K.: Cambridge Univ. Press, 2004.

[9] I. S. Gradshteyn and I. M. Ryzhik, Table of Integrals, Series and Products, 7th ed. Amsterdam, The Netherlands: Elsevier Academic, 2007.

[10] T. W. Anderson, An Introduction to Multivariate Statistical Analysis, 3rd ed. Cambridge, U.K.: Wiley-Interscience, 2003.

[11] T. M. Cover and J. A. Thomas, Elements of Information Theory. New York, NY, USA: Wiley, 2006.

[12] J. M. McNamee, Numerical Methods for Roots of Polynomials-Part 1. Amsterdam, The Netherlands: Elsevier Sci., 2007, vol. 14, ser. Stud. Comput. Math..

[13] S. Verdu, Multiuser Detection. Cambridge, U.K.: Cambridge Univ. Press, 1998.

[14] A. J. Goldsmith, Wireless Communications. Cambridge, U.K.: Cambridge Univ. Press, 2005.

[15] V. K. Rohatgi and A. K. Saleh, An Introduction to Probability and Statistics, 2nd ed. New York, NY, USA: Wiley, 2001, ser. Probability Statist.

[16] B. E. Meserve, Fundamental Concepts of Algebra. New York, NY, USA: Dover, 1953.

[17] M. Fujiwara, "Über die obere schranke des absoluten betrages der wurzeln einer algebraischen gleichung," Tôhoku Math. J., vol. 10, pp. $167-171,1916$.

[18] T. Kojima, "On the limits of the roots of an algebraic equation," Tôhoku Math. J., vol. 11, pp. 119-127, 1917.

[19] P. Batra, "A property of the nearly optimal root-bound," J. Comp. App. Math., vol. 167, no. 2, pp. 489-491, 2004.

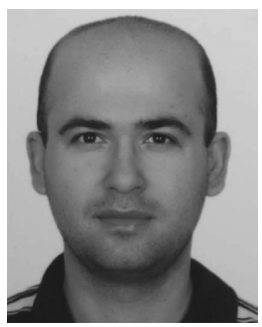

Berkan Dulek (S'11-M'13) received the B.S., M.S. and $\mathrm{Ph} . \mathrm{D}$. degrees in electrical and electronics engineering from Bilkent University in 2003, 2006, and 2012, respectively. From 2007 to 2010, he worked at Tübitak Bilgem İltaren Research and Development Group. From 2012 to 2013, he was a Postdoctoral Research Associate with the Department of Electrical Engineering and Computer Science, Syracuse University, Syracuse, NY, USA. His research interests are in statistical signal processing, detection and estimation theory, and wireless communications. 